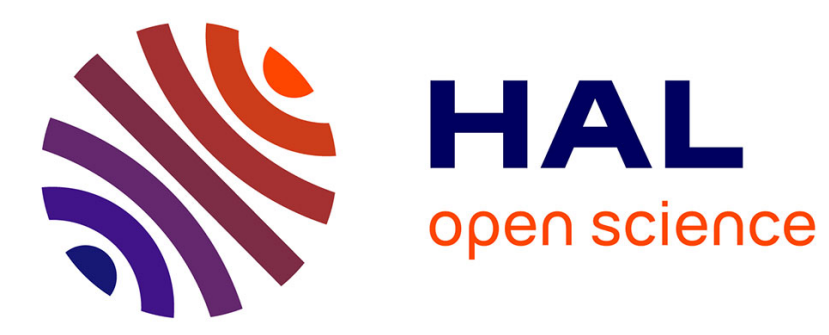

\title{
Birefringent distributions tailored for imaging and other applications
}

\author{
Miguel Alonso, Anthony Vella
}

\section{To cite this version:}

Miguel Alonso, Anthony Vella. Birefringent distributions tailored for imaging and other applications. 2018 17th Workshop on Information Optics (WIO), Jul 2018, Québec, Canada. pp.1-3. hal-02024696

\section{HAL Id: hal-02024696 https://hal.science/hal-02024696}

Submitted on 27 Feb 2019

HAL is a multi-disciplinary open access archive for the deposit and dissemination of scientific research documents, whether they are published or not. The documents may come from teaching and research institutions in France or abroad, or from public or private research centers.
L'archive ouverte pluridisciplinaire HAL, est destinée au dépôt et à la diffusion de documents scientifiques de niveau recherche, publiés ou non, émanant des établissements d'enseignement et de recherche français ou étrangers, des laboratoires publics ou privés. 


\section{Birefringent distributions tailored for imaging and other applications}

\author{
$1^{\text {st }}$ Miguel A. Alonso \\ Aix Marseille Univ., CNRS, Centrale Marseille, Institut Fresnel \\ UMR 7249, 13397 Marseille Cedex 20, France \\ The Institute of Optics, \\ Center for Coherence and Quantum Optics \\ University of Rochester \\ Rochester NY 14627, U.S.A. \\ miguel.alonso@fresnel.fr
}

\author{
$2^{\text {nd }}$ Anthony Vella \\ The Institute of Optics \\ Center for Coherence and Quantum Optics \\ University of Rochester \\ Rochester NY 14627, U.S.A. \\ avella@ur.rochester.edu
}

\begin{abstract}
Spatially-varying birefringent plates such as metasurfaces, q-plates, and stressed-engineered windows have received considerable attention in recent years. Here, a general geometric representation of birefringence is presented. Several specific applications are also considered, corresponding to imaging polarimetry, microscopy, and structured light generation.

Index Terms-birefringence, polarization, structured light, geometric phase, imaging
\end{abstract}

\section{INTRODUCTION}

Spatially-varying birefringence in optical components occurs naturally due to internal mechanical stress, and is often regarded as detrimental to the performance of a system. However, some applications benefit from using elements with tailored spatially-varying birefringence, and several technologies have been proposed for implementing these elements, including metasurfaces [1], liquid crystal devices such as q-plates [2], d-plates [3] and light valves [4], as well as stress-engineered birefringence [5]. In this work, we discuss a geometric description of spatially-varying birefringence distributions [6] based on a generalization of the Poincaré sphere geometric construction, which is usually employed to describe beam polarization and not birefringent elements.

\section{LOCAL BIREFRINGENCE}

We consider elements in the form of transparent masks, intended for use at (near) normal incidence. At each point, these masks have two orthonormal eigenpolarizations, that is, polarizations that do not change upon transmission. When light with arbitrary polarization is incident on the mask, these two polarization components acquire different phases, $\Gamma+\delta$ and $\Gamma-\delta$, so that the relative phase retardance between them is $2 \delta$. The slow eigenpolarization can be characterized by the latitude and longitude angles, $\Theta$ and $\Phi$, over the Poincare sphere; the fast eigenpolarization corresponds to the antipodal coordinates, namely $-\Theta$ and $\Phi+\pi$.

National Science Foundation (NSF) (PHY-1507278). Excellence Initiative of Aix-Marseille University - A* MIDEX, a French "Investissements d'Avenir" programme.

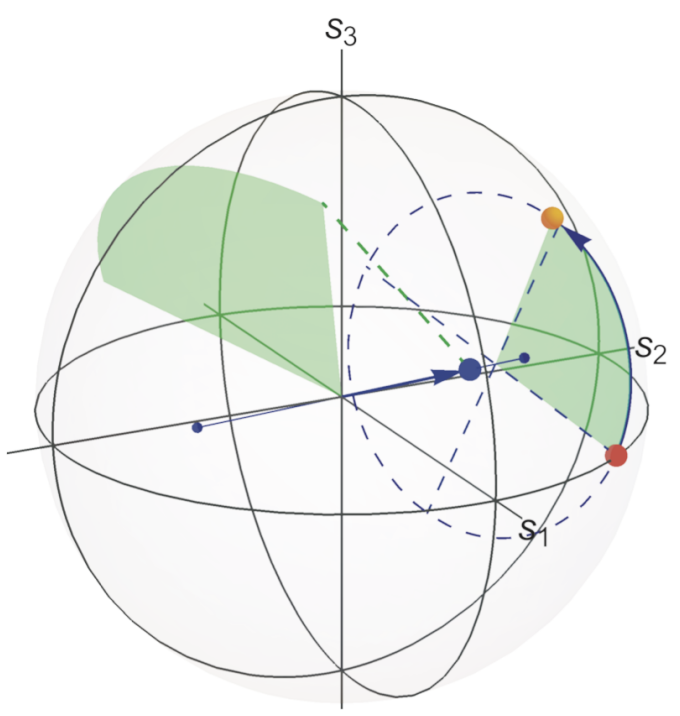

Fig. 1. Birefringence representation on the Poincaré sphere. The large blue dot represents the birefringence point $\mathbf{q}$, and the smaller two blue dots over the sphere's surface and colinear with this dot and the origin are the two eigenpolarizations. The red and orange dots represent, respectively, the input and output polarization states $\mathbf{s}$ and $\mathbf{s}^{\prime}$, the latter resulting from the rotation of the former around the line in the direction of $\mathbf{q}$ by an angle $2 \arcsin |\mathbf{q}|$, represented by the green fans.

The Jones matrix describing the local polarization transformation for an arbitrarily polarized incident beam is, in the circular polarization (or helicity) basis, given by

$$
\mathbb{J}=\exp (\mathrm{i} \Gamma)\left[\begin{array}{rr}
q_{0}-\mathrm{i} q_{3} & -q_{2}-\mathrm{i} q_{1} \\
q_{2}-\mathrm{i} q_{1} & q_{0}+\mathrm{i} q_{3}
\end{array}\right],
$$

where the quantities $q_{n}$ are defined as

$$
\begin{aligned}
& q_{0}=\cos \delta \\
& q_{1}=\sin \delta \cos \Theta \cos \Phi, \\
& q_{2}=\sin \delta \cos \Theta \sin \Phi, \\
& q_{3}=\sin \delta \sin \Theta .
\end{aligned}
$$


Notice that these four quantities can be interpreted as the components of a four-dimensional unit vector $\vec{q}=\left(q_{0}, q_{1}, q_{2}, q_{3}\right)$. That is, if the global phase $\Gamma$ is ignored, the birefringence can be represented as a point over a four-dimensional unit hypersphere, just as (full) polarization corresponds to a point over the unit three-dimensional Poincaré sphere. Further, because a phase delay $2 \delta>\pi$ is equivalent to a phase delay $2 \pi-2 \delta$ with the eigenpolarizations exchanged, it is sufficient to consider $\delta \in[0, \pi / 2]$, i.e., only half of the hypersphere is needed. This means that $q_{0}$ can be inferred uniquely from the other three coordinates, so birefringence can be described uniquely by the three-vector $\mathbf{q}=\left(q_{1}, q_{2}, q_{3}\right)$. The geometric interpretation of this vector is simple: if light with a polarization characterized by a point $\mathbf{s}$ over the Poincaré sphere is incident on the birefringent element, the transmitted light has polarization $\mathbf{s}^{\prime}$ which corresponds to a rotation of $\mathbf{s}$ around the direction of $\mathbf{q}$ by an angle $2 \arcsin |\mathbf{q}|$ according to the right-hand rule (see Fig. 1).

\section{GEOMETRIC PHASE}

Suppose that two birefringent masks, characterized respectively by vectors $\mathbf{q}_{\mathrm{a}}$ and $\mathbf{q}_{\mathrm{b}}$, are concatenated. The resulting composite element is then characterized by the vector $\mathbf{q}_{\mathrm{ab}}=\mathbf{q}_{\mathrm{a}} \sqrt{1-\left|\mathbf{q}_{\mathrm{b}}\right|^{2}}+\mathbf{q}_{\mathrm{b}} \sqrt{1-\left|\mathbf{q}_{\mathrm{a}}\right|^{2}}+\mathbf{q}_{\mathrm{a}} \times \mathbf{q}_{\mathrm{b}}$, where the last term accounts for the noncommutability of the elements. This composite element also has two eigenpolarizations, corresponding to the points $\pm \mathbf{q}_{\mathrm{ab}} /\left|\mathbf{q}_{\mathrm{ab}}\right|$ over the Poincaré sphere, and with a phase delay between them of $2 \arcsin \left|\mathbf{q}_{a b}\right|$. The phase acquired by each of these polarizations is composed not only of the dynamic phases for each, given by the sum of the corresponding phases, $\Gamma_{\mathrm{a}}+\Gamma_{\mathrm{b}}$, but also of a geometric phase related to the path traced by the polarization over the Poincare sphere. Unlike other occurrences of geometric phase in nature for which the paths in question over the Poincaré sphere follow what is known as parallel transport, this geometric phase is in general not given by one half of the enclosed solid angle [7], [8]. It does, however, have the simple geometric interpretation over the Poincaré sphere, as shown in Fig. 2: it equals the angle between the projections of $\mathbf{q}_{\mathrm{a}}$ and $\mathbf{q}_{\mathrm{b}}$ onto a plane normal to either of the eigenpolarizations [6]. Note that this interpretation is different but fully equivalent to that given by Courtial [9], [10].

\section{SPATIALLY-VARYING BIREFRINGENCE AND APPLICATIONS}

The description given so far corresponds to the birefringence at a single point of the birefringent element (or the concatenation of several of them). When considering spatially-varying birefringent elements, one can map the transverse coordinates $\mathbf{x}$ onto points $\mathbf{q}(\mathbf{x})$, so that the element is characterized by a surface inside the Poincaré sphere. Figure 3 shows three examples. This representation is useful for understanding the effects of spatially-varying birefringence on the performance of an optical system. For example, simple geometric interpretations in terms of the Poincare hypersphere were given to both the widening of the PSF and the reduction of the Strehl ratio

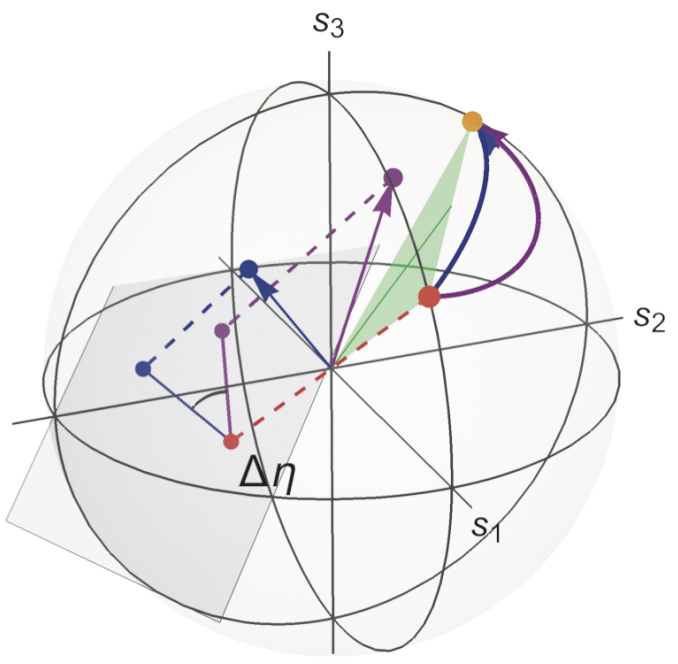

Fig. 2. Geometric interpretation of the geometric phase, following the transformation of an initial polarization (red dot) to a new polarization (orange dot) through a birefringent element characterized by $\mathbf{q}_{\mathrm{a}}$ (purple dot and arrow), and then back to the initial polarization by a second birefringent element characterized by $\mathbf{q}_{\mathrm{b}}$ (blue dot and arrow). The geometric phase corresponds to the angle $\Delta \eta$ between the projections of the birefringence axes $\mathbf{q}_{\mathrm{a}}$ and $\mathbf{q}_{\mathrm{a}}$ onto a plane normal to the line from the origin to the (red) point representing the input polarization.

of imaging systems, due to spatially-varying birefringence of its elements [6]. This formalism has also been used for the design of birefringence distributions that are optimal for different goals, such as the generation of bottle beams [11] and of polarimetric systems [12]. In both cases, the optimal distributions have been found to be extremely similar to the stable birefringence pattern that results from subjecting a glass window to a pressure distribution with trigonal symmetry at the edges [5]. These so-called stress-engineered optics (SEOs) have been applied already for generating beams with interesting polarization distributions [13], [14], imaging polarimetry [15]-[17] and bottle beam generation [11], and they are currently being considered for applications in microscopy [18].

\section{ACKNOWLEDGMENT}

We thank our collaborators, past and present, on topics related to spatially-varying birefringence: Thomas G. Brown, Amber Beckley, Roshita Ramkhalawon, Ashan Ariyawansa, Luis A. Alemán-Castaneda, Sophie Brasselet, Hervé Rigneault, Philippe Réfrégier, Valentina Curcio, Valentine Wasik, Lukas Novotny, Hyppolite Dourdent, Lorenzo Marrucci, Enrico Santamato, and Bruno Piccirillo.

\section{REFERENCES}

[1] A. Arbabi, Y. Horie, M. Bagheri, and A. Faraon, "Dielectric metasurfaces for complete control of phase and polarization with subwavelength spatial resolution and high transmission," Nat. Nanotechnol. vol. 10, pp. 937-943, 2015.

[2] L. Marrucci, "The q-plate and its future," J. Nanophot. vol. 7, pp. 078598-078598, 2013. 

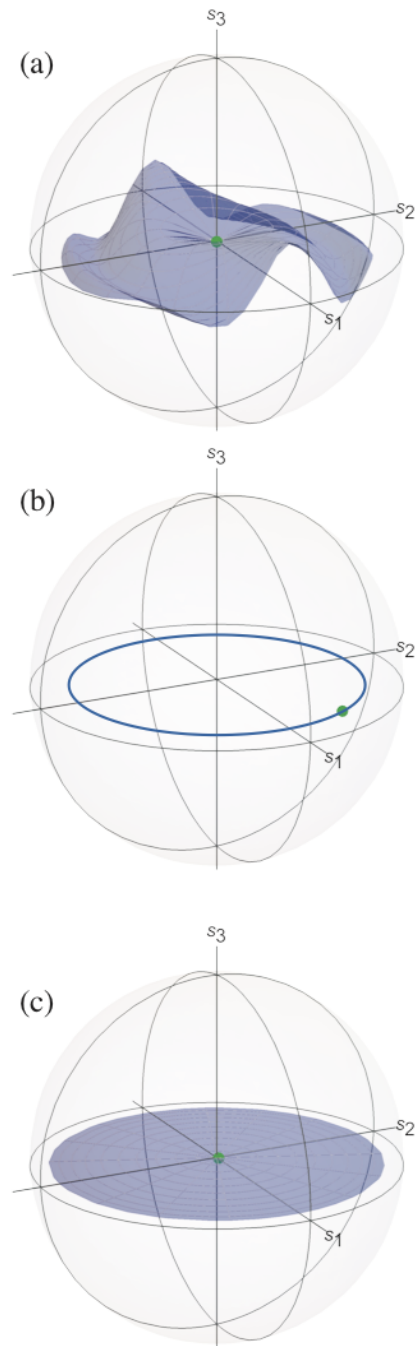

Fig. 3. Poincaré sphere representation of three spatially-varying birefringent elements: (a) a generic element that includes elliptic eigenpolarizations, (b) a q-plate, for which the surface collapses to a circle whose radius is the sine of half the retardance, and (c) an SEO.

[3] L.A. Alemán-Castaneda, B. Piccirillo, E. Santamato, L. Marrucci, and M.A. Alonso, "Wavefront sensing via variable scale shearing interferometry based on geometric phases," in preparation.

[4] E. Brasselet, "Tunable high-resolution macroscopic self-engineered geometric phase optical elements," Phys. Rev. Lett., vol. 121, 033901, 2018.

[5] A. K. Spilman and T. G. Brown, "Stress birefringent, space-variant wave plates for vortex illumination", Appl. Opt., vol. 26, pp. 61-66, 2007.

[6] A. Vella and M.A. Alonso, "Poincaré sphere representation for spatially varying birefringence," Opt. Lett., vol. 43, pp. 379-382, 2018.

[7] S. Pancharatnam, "Generalized theory of interference and its applications. Part I. Coherent pencils," Proc. Indian Acad. Sci. A, vol. 44, pp. 247-262, 1956.

[8] M. V. Berry, "The adiabatic phase and Pancharatnam's phase for polarized light," J. Mod. Opt., vol. 34, pp. 1401-1407, 1987.

[9] J. Courtial, "Wave plates and the Pancharatnam phase," Opt. Communn., vol. 171, pp. 179-183, 1999.

[10] P. Kurzynowski, W.A. Woźniak, and M. Szarycz, "Geometric phase: two triangles on the Poincaré sphere," J. Opt. Soc. Am. A, vol. 28, pp. 475-482, 2011.

[11] A. Vella, H. Dourdent, L. Novotny, and M.A. Alonso, "Birefringent masks that are optimal for generating bottle fields," Opt. Express, vol. 25, pp. 9318-9332, 2017; Erratum, vol. 25, p. 19654, 2017.

[12] A. Vella, T.G. Brown, and M.A. Alonso, 'Optimal birefringence distributions for imaging polarimetry," in preparation.

[13] A.M. Beckley, T.G. Brown, and M.A. Alonso, "Full Poincaré beams," Opt. Express, vol. 18, pp. 10777-10785, 2010.

[14] A.M. Beckley, T.G. Brown, and M.A. Alonso, "Full Poincaré beams II: Partial polarization," Opt. Express, vol. 20, pp. 9357-9362, 2012.

[15] R. Ramkhalawon, T.G. Brown, and M.A. Alonso, "Imaging the polarization of a light field," Opt. Express, vol. 21, pp. 4106-4115, 2013.

[16] B.G. Zimmerman and T.G. Brown, "Star test image-sampling polarimeter," Opt. Express, vol. 24, pp. 23154-23161, 2016.

[17] S. Sivankutti, E.R. Andresen, G. Bowmans, T.G. Brown, M.A. Alonso, and H. Rigneault, "Single shot polarimetry of multicore fiber," Opt. Lett., vol. 41, pp. 2105-2108, 2016.

[18] V. Curcio, T.G. Brown, S. Brasselet, and M.A. Alonso, "Simultaneous determination of $3 \mathrm{D}$ orientation and $3 \mathrm{D}$ localization in single emitter microscopy imaging," in preparation. 